\title{
Non-Rigid Image Registration using Bone Growth Model
}

\author{
Morten Bro-Nielsen ${ }^{123}$, Claus Gramkow ${ }^{23}$, Sven Kreiborg ${ }^{2}$ \\ ${ }^{1}$ HT Medical, Inc., 6001 Montrose Road, Suite 902, Rockville, MD 20852, USA \\ ${ }^{2} 3 \mathrm{D}-\mathrm{Lab}$, School of Dentistry, Univ. of Copenhagen, Denmark \\ ${ }^{3}$ Dept. of Mathematical Modelling, Technical University of Denmark \\ e-mail: bro@ht.com WWW http://www.imm.dtu.dk/ ${ }^{\sim}$ bro
}

\begin{abstract}
Non-rigid registration has traditionally used physical models like elasticity and fluids. These models are very seldom valid models of the difference between the registered images. This paper presents a non-rigid registration algorithm, which uses a model of bone growth as a model of the change between time sequence images of the human mandible. By being able to register the images, this paper at the same time contributes to the validation of the growth model, which is based on the currently available medical theories and knowledge.
\end{abstract}

This paper studies the process of bone growth in the human mandible. Based on the available medical knowledge, a bone growth model is established, and experiments are carried out to determine the validity of the model. These experiments uses a unique data set containing three voxel images of the mandible of a child, taken at different times.

Being able to model the growth of the facial bones is both interesting for understanding the development of the cranio-facial complex, and for understanding the effects of cranio-facial surgery on children. After children with growth defects have undergone surgery to restore normal function and appearance, the bones continue to grow. This growth often introduces new deformations and new surgery has to be performed.

It is, therefore, important to understand the growth of the bones. It would be useful for the surgeon to be able, not only to predict the instantaneous result of the surgery, but also to predict the evolution of the face after surgery. Using such knowledge, the surgeon could possibly anticipate this development and correct for it in advance.

The process of modeling the growth can be seen as a registration problem, and the discussion follows the notation and semantics of the general registration methodology.

\section{Non-rigid registration as a physical problem}

Non-rigid registration is used to register images that are different. As 'non-rigid' indicates, more than just a simple superposition of the images is required to get 
a complete correspondence, and some sort of deformation must be included in the transformation, to map dissimilar images onto each other.

Optimally, the non-rigidness of the transformation allows a full correspondence to be determined, and at the same time limit excessive deformations by including some sort of regularization effect.

Most non-rigid registration methods are directly or indirectly based on elastic (eg. $[1,12]$ or viscous-fluid models (eg. [9, 8]). Although many methods do not use the terminology of these continuum models, they follow the same ideas algorithmicly.

The use of elastic and viscous fluid models for registration of images has often been criticized, because they represent the application of a physical model, which does not reflect the actual physical development between the images.

A correct physical model, for registration of images of the brain of two individuals, would reflect the actual biologic and genetic development of these brains. Since a common genetic basis exists for these brains, it should, conceptually, be possible to create a development model for the two brains down through history to a common ancestor. Using this development model a correspondence could be created.
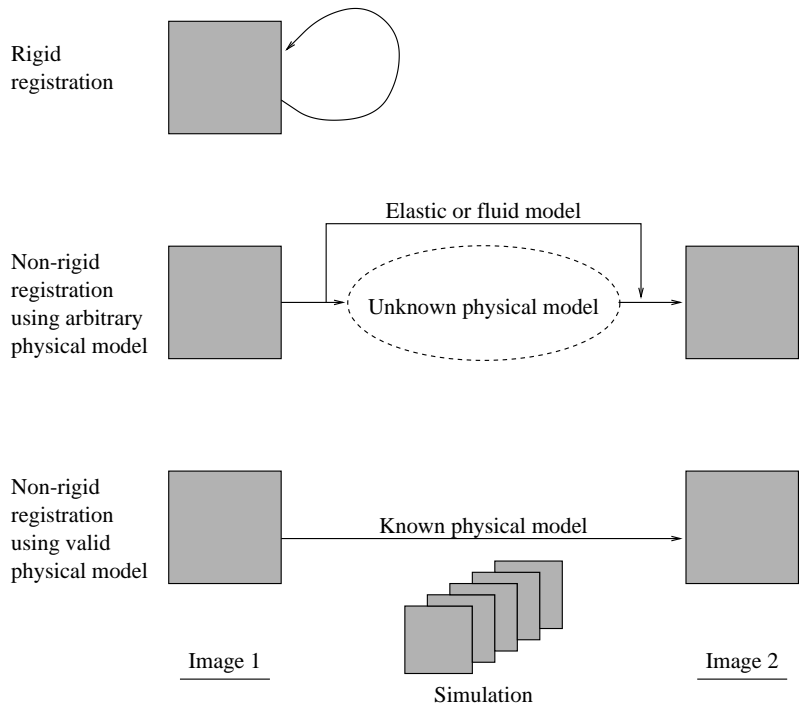

Fig. 1. Different registration methods and their use of physically valid or invalid models of the change between two images.

The difference between the traditional registration models and the idea of a physically correct model of development, is illustrated in figure 1. Rigid registration methods basicly register images to derivatives of themselves. The traditional non-rigid registration methods attempt to model the difference between two im- 
ages, using an arbitrary physical model, which does not reflect the actual physical process. The development methods, on the other hand, use the actual physical process as the model of change.

One of the important advantages, of using a physically correct model, is the ability to simulate what happens between the images. Where traditional nonrigid methods only provide a correspondence between the images, the physically valid models describe the actual development, which can then be simulated. Naturally, these models have generally been viewed as impossible, although conceptually interesting.

In this paper a physically valid development model, for non-rigid registration of time sequence images of the human mandible is developed. With a model of physical bone growth, images are registered by allowing the initial image to grow to the second image. The initial and final images are used as boundary conditions.

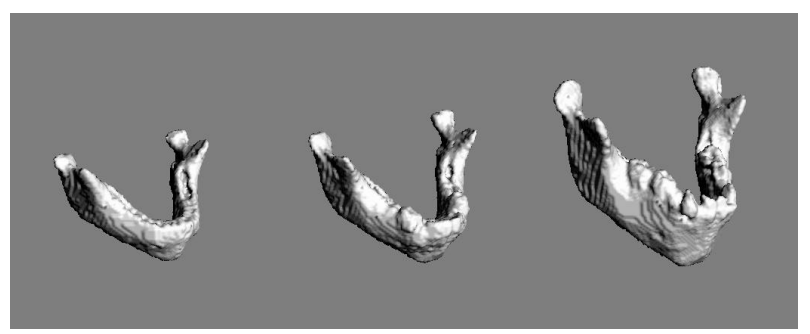

Fig. 2. Images of the mandible of a child at the age of 9,21 months and 7 years.

A set of three images of the mandible of the same child at different times, is used to validate the growth model (see figure 2). These images have been acquired at the age of 9,21 months and 7 years.

During this period both actual bone growth, and teeth growth and eruption take place. But for simplicity, we ignore the teeth growth and consider the mandible as one coherent piece of bone. The teeth and other internal structures are only used for initial rigid registration of the images.

\section{Bone growth}

A general rule for bone growth, is that growth only takes place on the surface of bones. The interior of bones is calcified and rigid, and do not change shape [10, 11]. Bone growth is consequently different from normal biological growth, where growth happens by cell division.

Bones grow by a complex process of deposition ${ }^{1}$ and resorption. Deposition adds new material to the surface of the bone and resorption removes material

\footnotetext{
${ }^{1}$ Sometimes called apposition.
} 
from the surface of the bone. These processes only occur in a thin boundary layer, in which cells called osteoblasts and osteoclasts are responsible for the deposition and resorption of bone material respectively.

Deposition can take place both on the surface of normal bone, in which case it is called surface deposition, in sutures where bones are connected by thin bands of fibrous material, and in the cartilages that join some bones. Examples of sutures are found in the skull, where they accommodate the growth of the brain, and examples of cartilages are found on the condyles of the mandible, where they form the joint between the mandible and the skull.

Surface deposition is considerably slower than the other forms of growth, but takes place on the entire surface. Cartilage and suture growth only take place in isolated areas.

\subsection{Growth of the mandible}

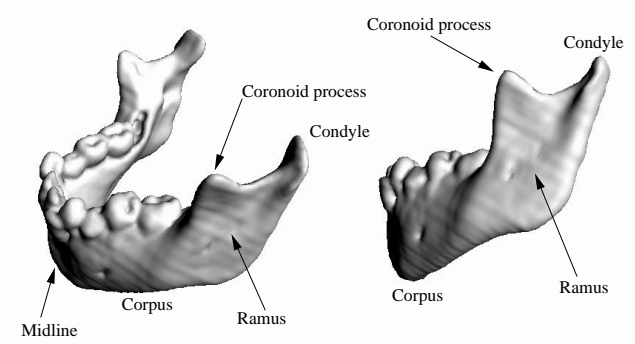

Fig. 3. Anatomic description of the mandible.

The general anatomy of the mandible is shown in figure 3 .

Up to the second half of the first year the mandible has cartilage in the midline. But after this period the two halves are connected and the cartilage disappears. The mandible is then a single bone, with cartilages on the two condyles (the mandibular condylar cartilages).

Growth of the mandible takes place by growth in the condylar cartilages, and by surface deposition and resorption on the remaining surface of the mandible.

That the mandible only grows by surface processes can seem surprising, considering the large size difference between the jaw of a child and an adult. But implant studies by Björk [3, 4] and Björk and Skieller [6, 7] have shown this model of the growth process to be valid at least for the corpus. The growth pattern of the condyles is still under discussion.

\section{$2.2 \quad$ Stable structures}

Since the interior of the mandible does not grow, it should be expected that stable structures could be detected here. 


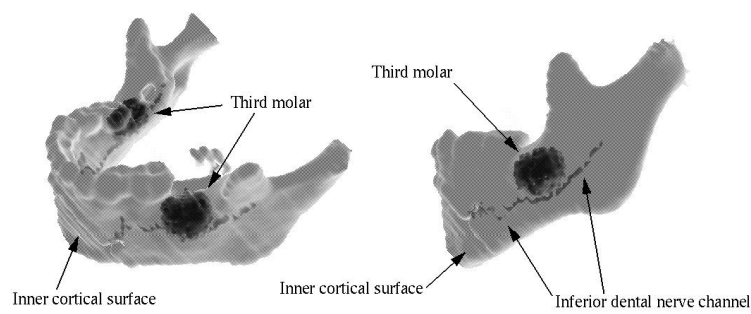

Fig. 4. Stable features in the mandible.

Indeed based on his implant studies, Björk [5] suggested the inferior dental nerve channel, the inner cortical surface of the symphysis, the lower margin of the third molar tooth germ before it erupts, and the tip of the chin as stable features of the mandible (see figure 4 ).

There is general agreement on these features, although the third molar is only stable during the period, in which it has not started developing roots. It can, therefore, not be used in studies over many years.

\section{Non-rigid registration of mandibles using bone growth model}

Using the information about the growth of the mandible, this section establishes a mathematical model of the growth process, and a simulation algorithm, which allows non-rigid registration of time sequence images of a developing jaw and, thereby, simulation of its development.

The basic algorithm first rigidly registers the mandibles using the stable structures suggested by Björk [5]. When these structures have been overlaid, the remaining difference between the images reflects the growth that has taken place between their acquisition.

A partial differential equation for the growth on the surface of the bone is set up, using the estimated distance between corresponding features to determine the growth velocity.

Finally, the growth process is simulated to register the images.

\subsection{Rigid registration of stable structures}

A combination of surface based rigid registration, based on the Iterated Closest Point (ICP) algorithm by Besl and Kay [2], and manual correction, were applied to register the mandibles. Using the ICP algorithm, the tip of the chin was first registered and the result subsequently validated and corrected using the other stable structures. Figure 5 shows the three mandibles after rigid registration. 


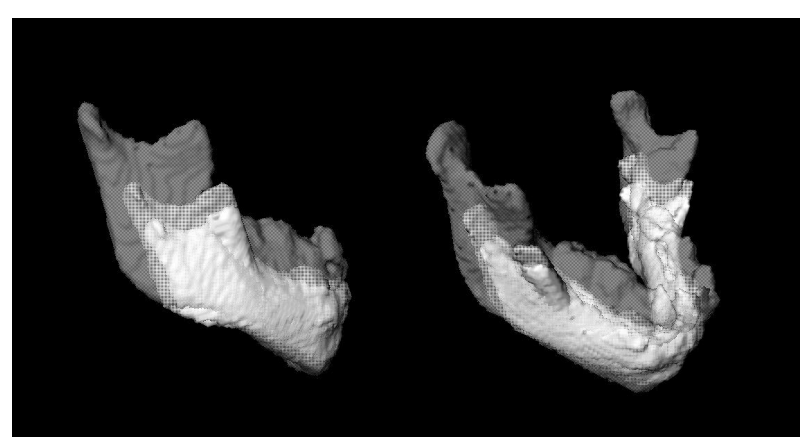

Fig. 5. Overlayed mandibles after rigid registration.

From the overlapping mandibles it is possible to discern some of the changes caused by the growth. The most dramatic growth takes place on the ramus, which is the large structure at the end of the mandible, and the condyles, which are the attached to the rami and serve as the connection to the rest of the skull.

\subsection{Growth model}

As we saw above, the deposition and resorption processes work in a thin boundary layer on the surface of the mandibles. During the work of the osteoblast and osteclast cells, the density of the bone increases or decreases in the boundary layer until bone has either been completely deposited or resorped. The boundary layer then moves, and the process restarts.

To model this, the bone density $\rho(\boldsymbol{x}, t) \in[0 ; 1]$ is used as the primary variable. Bone is defined as $\rho(\boldsymbol{x}, t)=1$ and air is defined as $\rho(\boldsymbol{x}, t)=0$. Thus, the definition of the bone body becomes $\Omega=\{\boldsymbol{x} \mid \rho(\boldsymbol{x}, t)=1\}$. We define the initial image as the template image $T(\boldsymbol{x}) \in[0 ; 1]$ and the desired end-state as the study image $S(\boldsymbol{x}) \in[0 ; 1]$. The initial domain can therefore be written as $\Omega=\{\boldsymbol{x} \mid \rho(\boldsymbol{x}, 0)=$ $T(\boldsymbol{x})\}$. The registration problem is formulated as changing the density map using a model of growth, so that $\rho\left(\boldsymbol{x}, t_{\text {end }}\right)=S(\boldsymbol{x})$ where $t_{\text {end }}$ is the time of full registration.

The boundary of the domain is denoted $\tilde{\Gamma}$ (see figure 6.6), and consists of a stable part $\tilde{\Gamma}_{0}$, where the template and study boundaries match, and unstable parts $\tilde{\Gamma}_{1}$, and $\tilde{\Gamma}_{-1}$ where deposition and resortion take place respectively:

$$
\begin{aligned}
\tilde{\Gamma} & =\tilde{\Gamma}_{0}+\tilde{\Gamma}_{1}+\tilde{\Gamma}_{-1} \\
\tilde{\Gamma}_{0} & =\{\boldsymbol{x} \in \tilde{\Gamma} \mid T(\boldsymbol{x})=S(\boldsymbol{x})\} \\
\tilde{\Gamma}_{1} & =\{\boldsymbol{x} \in \tilde{\Gamma} \mid T(\boldsymbol{x})<S(\boldsymbol{x}) \wedge \rho(\boldsymbol{x}, t) \in[0 ; 1[\} \\
\tilde{\Gamma}_{-1} & =\{\boldsymbol{x} \in \tilde{\Gamma} \mid T(\boldsymbol{x})>S(\boldsymbol{x}) \wedge \rho(\boldsymbol{x}, t) \in] 0 ; 1]\}
\end{aligned}
$$

For completeness we define the boundary as having a thickness $\delta>0$.

We now proceed to define the partial differential equation (PDE) governing the growth process. The general requirements for this PDE is that change in 
density should happen only for $\boldsymbol{x} \in \tilde{\Gamma}_{1}+\tilde{\Gamma}_{-1}$, and that the change in density should reflect the velocity of the actual growth. The PDE is defined as:

$$
\frac{\partial \rho(\boldsymbol{x}, t)}{\partial t}= \begin{cases}c v_{g}(\boldsymbol{x}, t) & x \in \tilde{\Gamma}_{1} \\ -c v_{g}(\boldsymbol{x}, t) & x \in \tilde{\Gamma}_{-1} \\ 0 & x \in \tilde{\Gamma}_{0} \cup \tilde{\Omega}\end{cases}
$$

where $v_{g}(\boldsymbol{x}, t)$ is the growth velocity and $c$ is a constant. The equation is conceptually simple, and states that the density should increase in areas of deposition, and decrease in areas of resorption. The rate of change is proportional to the growth velocity.
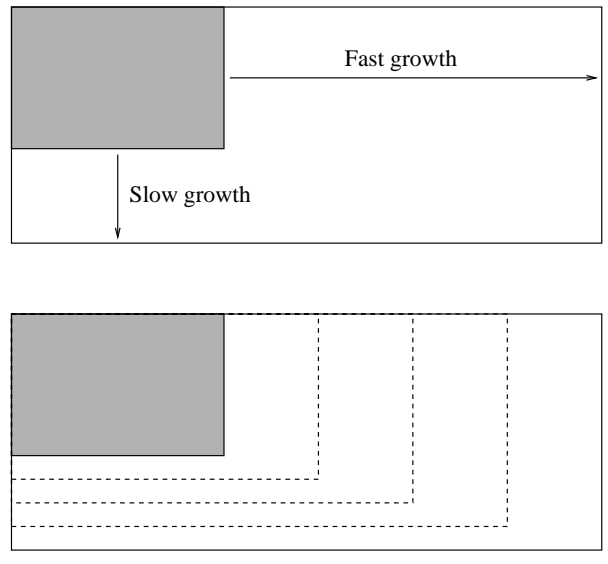
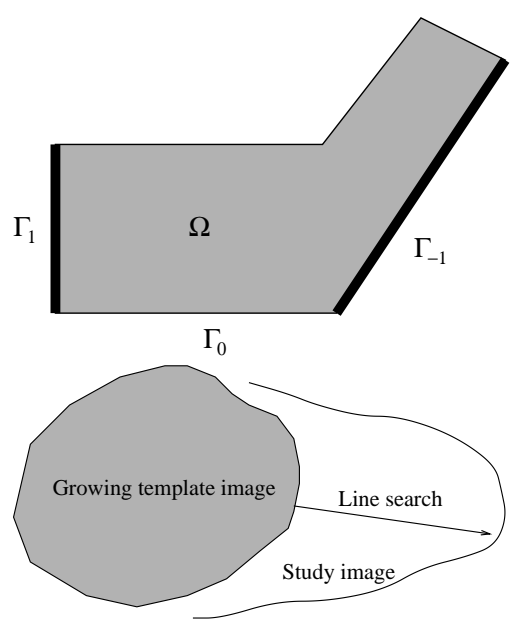

Fig. 6. Left: The speed of growth must be depend on the distance to be filled. Top right: Definition of growth domain. Lower right: Line process.

Determining the growth velocity A major problem, in developing the growth registration algorithm, is the selection of an appropriate growth velocity. The initial suggestion is a constant.

Consider figure 6. As this drawing illustrates, for the growth to look normal, it is necessary that the growth velocity reflects the distance, which needs to be covered by the process. Otherwise, the growth would first fill out the thin spaces, and then gradually fill out the wide spaces, thus falsely indicating that the thin spaces only grow in the beginning of the process.

Instead of a constant, the actual remaining distance from the boundary of the current bone body to the boundary of the study image $S(\boldsymbol{x})$, could be used as the velocity. But this does not work either since the velocity would decrease as the gap was narrowed. The growth velocity must reflect the initial distance from the template image boundary to the study image boundary. 
The suggested growth velocity is therefore determined using the following algorithm:

1. Initially, determine a distance map $\operatorname{dist}(\boldsymbol{x})$ from the study image $S(\boldsymbol{x})$ to the template image $T(\boldsymbol{x})$.

2. For each boundary voxel $\boldsymbol{x}_{b} \in \tilde{\Gamma}_{1}+\tilde{\Gamma}_{-1}$ in the currently evolved image:

(a) Perform a line search, perpendicular to the boundary starting from the current bone body $\tilde{\Omega}$, until a boundary voxel $\boldsymbol{x}_{s}$ in the study image $S(\boldsymbol{x})$ is found (see figure 6).

(b) Set the growth velocity $v_{g}\left(\boldsymbol{x}_{b}, t\right)$ to $\operatorname{dist}\left(\boldsymbol{x}_{s}\right)$.

3. Lowpass filter the velocity field using a Gaussian kernel.

The last action has been introduced to smooth discontinuities in the velocity field, and to remove spikes.

\subsection{Implementation}

The complete growth registration is implemented with the following iterative algorithm:

1. Determine a distance map dist $(\boldsymbol{x})$ from the study image $S(\boldsymbol{x})$ to the template image $T(\boldsymbol{x})$,

2. Determine the correct boundary $\tilde{\Gamma}=\tilde{\Gamma}_{0}+\tilde{\Gamma}_{1}+\tilde{\Gamma}_{-1}$ of the bone body $\tilde{\Omega}$.

3 . For all voxels on the changing boundary $\tilde{\Gamma}_{1}+\tilde{\Gamma}_{-1}$ determine the growth velocity using the algorithm above,

4. Calculate $\partial \rho / \partial t$ using equation 2 ,

5. Perform time integration of the density:

$$
\rho(\boldsymbol{x}, t)=\rho(\boldsymbol{x}, t)+\triangle \frac{\partial \rho(\boldsymbol{x}, t)}{\partial t}
$$

where $\triangle$ is the time step.

6. Where the density $\rho(\boldsymbol{x}, t)>1$ or $\rho(\boldsymbol{x}, t)<0$, disperse the excess equally to neighbouring boundary voxels.

7. If $\rho(\boldsymbol{x}, t)$ has changed, go to 2

The dispersion of excessive density in step 6 is necessary because of the final size of the time step.

\subsection{Results}

A 2D example is used to illustrate the effect of the choice of non-constant growth velocity. Figure 7 shows the template and study images and their differences. As can be seen in the figure, both deposition and resorption is necessary during the growth process.

Figure 7 shows the growth pattern for growth with constant growth velocity and distance dependent growth velocity. The image in the bottom right of both figures shows the development pattern. Dark values indicate early growth and 


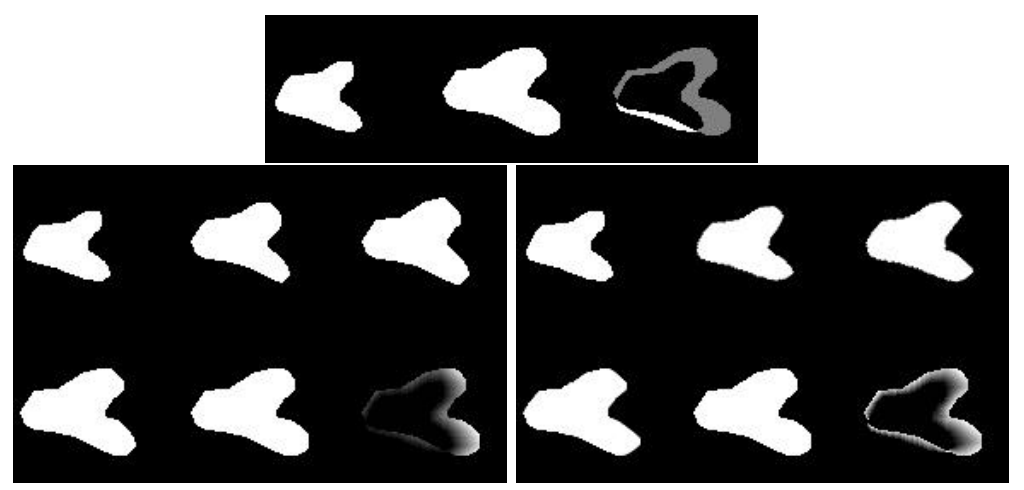

Fig. 7. Top: Initial image (left), image to grow to (middle), and the areas of differences (right). Bottom: Development of growth with simple velocity model (left) and distance dependent velocity model (right).

bright values late growth. It is easy to see how the growth, using the distance dependent growth velocity, has a more uniform development, whereas the pattern of the growth using constant velocity is more non-uniform.

The result of using the growth registration algorithm on the mandible images is shown in figure 8 . This figure shows that the development between the 3 original images is very smooth and looks quite realistic.

\section{Conclusion}

In this paper a model of bone growth in the human mandible has been developed. The results provide documentation that the theories of bone growth, which the model is based upon, may be correct. The documentation is not a definite proof, since it only shows that the theory can explain the growth of the mandible.

An important result of modeling the growth, is the time images, such as those in figure 7 , which are a by-product of the simulation. Together with corresponding images of the density change in each iteration, they convey important information about the structure of the growth. The clinical importance of these images has yet to be investigated.

This paper has argued that modeling the growth process, is an image registration process. As such, using the growth model as the basic control mechanism, represents a new form of medical image registration. All previous non-rigid registration algorithms have used physical models as regularization methods, and have not modeled the actual physical development responsible for the differences between the images.

\section{References}

1. R. Bajcsy and S. Kovacic, Multiresolution elastic matching, Computer Vision, Graphics and Image Processing, 46:1-21, 1989 


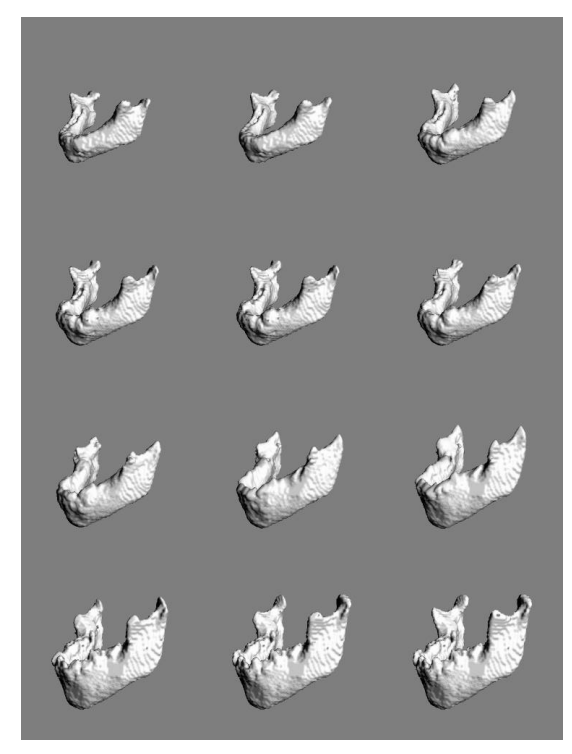

Fig. 8. Result of growth registration of 3 binary jaw images - top left, 2. row middle, and bottom right. The other images show intermediate results.

2. P.J. Besl and N.D. McKay, A methods for registration of 3-D shapes, IEEE Trans. Pattern Analysis and Machine Intelligence, 14(2):239256, 1992

3. A. Björk, The relationship of the jaws to the cranium, in A. Lundstrom, Introduction to orthodontics, McGraw-Hill, London, 1961

4. A. Björk, Variations in the growth pattern of the human mandible: Longitudinal radiographic study by the implant method, J. Dent. Res., 42:400, 1963

5. A. Björk, Prediction of mandibular growth rotation, Am. J. Ortho., 55:585, 1969

6. A. Björk and V. Skieller, Facial development and tooth eruption, Am. J. Ortho., $62: 339,1972$

7. A. Björk and V. Skieller, Growth of the maxilla in three dimensions as revealed radiographically by the implant method, Brit. J. Ortho., 4:53, 1977

8. M. Bro-Nielsen and C. Gramkow, Fast fluid registration of medical images, Proc. Visualization in Biomedical Imaging (VBC'96), pp. 267-276, 1996

9. G.E. Christensen, R.D. Rabbitt and M.I. Miller, 3D brain mapping using a deformable neuroanatomy, Physics in Medicine and Biology, 39:609-618, 1994

10. D.H. Goose and J. Appleton, Human dentofacial growth, Pergamon Press, ISBN 0-08-026393-3, 1982

11. F.P.G.M. Linden, Facial growth and facial orthopedics, Quintessence Publishing Co., Chicago, 1986

12. M.I. Miller, G.E. Christensen, Y. Amit and U. Grenander, Mathematical textbok of deformable neuroanatomies, Proc. Natl. Acad. Sci. USA, 90:1194411948, December 1993

This article was processed using the $\mathrm{AT}_{\mathrm{E}} \mathrm{X}$ macro package with LLNCS style 